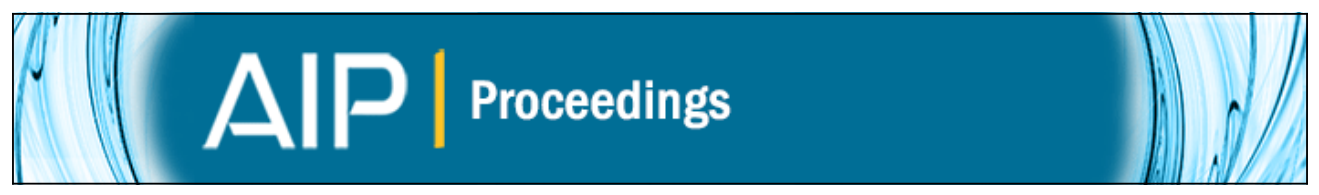

\title{
GD 358: The Case for Oblique Pulsation and Temperature Change
}

M. H. Montgomery

Citation: AIP Conference Proceedings 1273, 530 (2010); doi: 10.1063/1.3527882

View online: http://dx.doi.org/10.1063/1.3527882

View Table of Contents:

http://scitation.aip.org/content/aip/proceeding/aipcp/1273?ver=pdfcov

Published by the AIP Publishing

Articles you may be interested in

Searching for Planets with White Dwarf Pulsations: Spurious Detections

AIP Conf. Proc. 1331, 278 (2011); 10.1063/1.3556211

Limits of Perturbative Nonlinear Light Curve Analyses: the Case of G117-B15A AIP Conf. Proc. 1273, 512 (2010); 10.1063/1.3527877

GD 244: asteroseismology of a pulsator in the middle of the ZZ Ceti instability strip AIP Conf. Proc. 1273, 504 (2010); 10.1063/1.3527875

Post-main sequence evolution of debris discs

AIP Conf. Proc. 1273, 482 (2010); 10.1063/1.3527869

Swift observations of the Rapid Burster

AIP Conf. Proc. 1248, 195 (2010); 10.1063/1.3475201 


\title{
GD 358: The Case for Oblique Pulsation and Temperature Change
}

\author{
M. H. Montgomery \\ Department of Astronomy and McDonald Observatory, University of Texas, Austin, TX, USA
}

\begin{abstract}
We provide multiple lines of evidence that GD 358's convection zone was much thinner in 1996 than in 2006 and we interpret this as a result of a short-lived increase in its surface temperature. In addition, we find strong evidence of oblique pulsation using two sets of evenly split triplets in the 2006 data. This marks the first time that oblique pulsation has been identified in a variable white dwarf star as well as the first time that a temperature change has been observed in a single white dwarf.
\end{abstract}

Keywords: convection — stars: oscillations — stars: variables: general — stars: individual (GD 358)

PACS: $97.20 . \mathrm{Rp}, 97.30 .-\mathrm{b}$

\section{ASTROPHYSICAL CONTEXT}

The DBV GD 358 has been a leader in many respects. It was the first DBV to be discovered [1], it was the first DAV or DBV to have a well defined period spacing [2], and it was the first DAV or DBV to show clear evidence of triplets for many different radial overtones [3]. So it should perhaps come as no surprise that two more "firsts" have been added to the list: 1) first single white dwarf to show evidence of a large change in $T_{\text {eff }}$, and 2) first white dwarf to show evidence of oblique pulsation [4]. We examine these claims below.

\section{NONLINEAR LIGHT CURVE FITS}

Montgomery [5] demonstrated that by considering the nonlinear response of the convection zone $[6,7]$ one could obtain excellent fits to the light curves of two (nearly) single mode white dwarf pulsators. We have since extended this technique to multi-periodic stars [8] and have taken into account the nonlinear relationship between the bolometric and observed flux variations [9, 4]. Our approach is well-summarized in Montgomery [5] and Montgomery [9].

The principal equation we derive relates the local flux at a given $(\theta, \phi)$ entering the convection zone at its base, $F_{\text {base, }}$, and that leaving it at the photosphere, $F_{\text {phot }}$ :

$$
F_{\text {phot }}=F_{\text {base }}+\tau_{C} \frac{d F_{\text {phot }}}{d t},
$$

where the new timescale $\tau_{C} \equiv \tau_{C}\left(F_{\text {phot }}\right)$ describes the changing heat capacity of the convection zone as a function of the local photospheric flux. We parameterize it as

$$
\tau_{C}=\tau_{0}\left(\frac{T_{\mathrm{eff}}}{T_{\mathrm{eff}, 0}}\right)^{-N}
$$




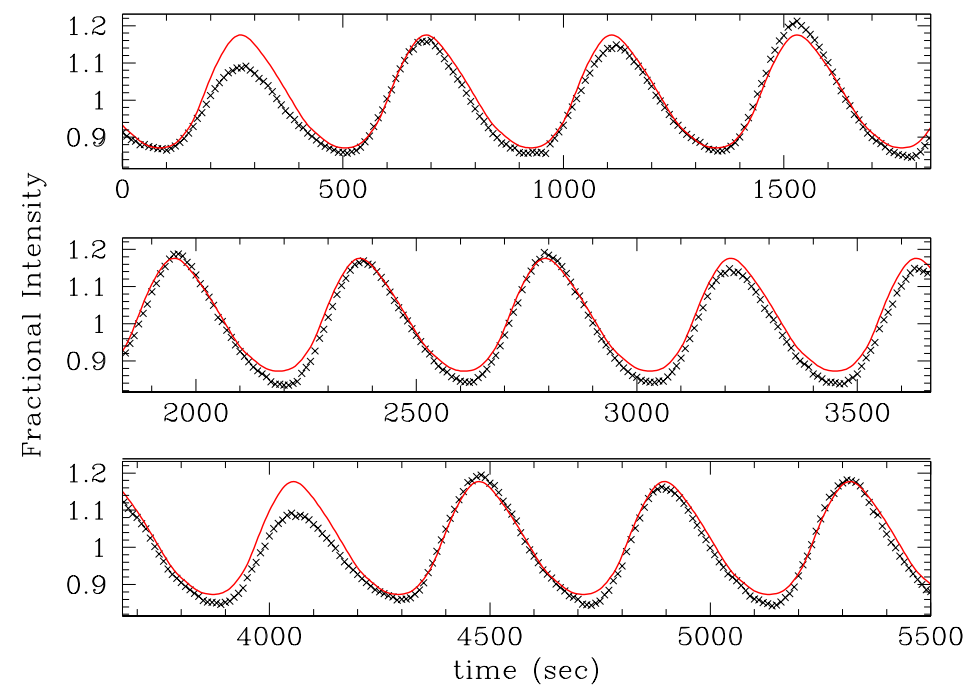

FIGURE 1. A section of the light curve of GD 358 during the sforzando event in 1996. The crosses are the data points and the curve is the best nonlinear light curve fit. From the fits $24<\tau_{0}<42 \mathrm{sec}$.

where $\tau_{0}$ is the equilibrium value of $\tau_{C}, T_{\text {eff }}$ is the instantaneous effective temperature and $T_{\text {eff, } 0}$ is its equilibrium value, and $N$ is a parameter describing the sensitivity of $\tau_{C}$ to changes in $T_{\text {eff. }}$. From standard mixing length theory of convection we expect that $N \sim 90$ for DAVs and $N \sim 23$ for DBVs. It is this extreme temperature sensitivity which is responsible for the large nonlinearities seen in white dwarf pulsations. We further assume that the angular dependence of $F_{\text {base }}$ is that of a spherical harmonic $Y_{\ell m}$, so we can use equation (1) to calculate the bolometric flux changes at the surface of the model. We then average over the visible disk of the model to obtain the theoretical light curves.

\section{EVIDENCE FOR TEMPERATURE CHANGE}

In 1996, within a period of $36 \mathrm{hrs,} \mathrm{all} \mathrm{of} \mathrm{the} \mathrm{power} \mathrm{at} \mathrm{periods}>700 \mathrm{~s}$ disappeared within detection limits. At the same time, GD 358 more than doubled its apparent pulsation amplitude, with power appearing almost exclusively at lower $k$, with a period of $\sim 420 \mathrm{~s}$. Over the next week, its amplitude decreased to "normal" levels, while the high $k$ power did not return for approximately one month. This dramatic change is documented in several papers, most recently in Kepler et al. [10] and Provencal et al. [11]. The episode itself is termed the "sforzando" after a musical term for a sudden and short-lived increase in loudness. From nonlinear light curve fits, such as that shown in Figure 1, we find that $\tau_{0}$ lies in the range of $24 \mathrm{~s}$ to $42 \mathrm{~s}$. We note that these values are consistent with the previous analysis of Weidner and Koester [12].

In contrast, during the May 2006 WET run GD 358 was in a "normal" state, as shown by the example light curve in Figure 2. Our simultaneous fit to a large subset 

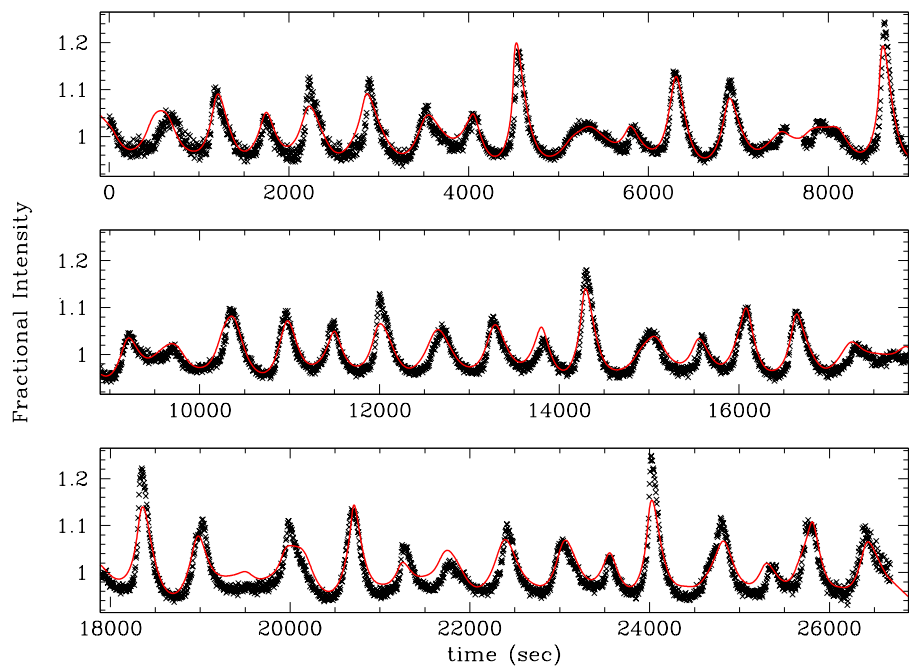

FIGURE 2. A comparison of the nonlinear light curve fit of the 2006 WET run (red curve) to data taken at McDonald Observatory on 23 May 2006 (crosses).

of the observed light curves yielded the following parameters: $\tau_{0}=586.0 \pm 11.7 \mathrm{~s}$, $N=27.4 \pm 0.7$, and $\theta_{i}=47.5 \pm 2.2^{\circ}$ [4]. Here, $\theta_{i}$ is the inclination angle of the pulsation axis to our line of sight.

This large change in $\tau_{0}$, from $\sim 590 \mathrm{~s}$ to $\sim 30 \mathrm{~s}$, can be explained in terms of a temperature change of the surface layers of GD 358: a higher $T_{\text {eff }}$ star should have a thinner convection zone and thus a correspondingly smaller time scale $\tau_{0}$. Using standard mixing length theory, the inferred temperature change is $\sim 2000 \mathrm{~K}$. While an interesting explanation, is there independent evidence for a change in GD 358's temperature?

Fortunately, there is. As shown in Figure 3, data from two sites in 1996 showed that the average intensity of GD 358 changed during the "sforzando". Taken together and with the evidence from the nonlinear light curve fits, this offers convincing evidence for a short-lived temperature change in the surface layers of GD 358.

\section{THE CASE FOR OBLIQUE PULSATION}

The multiplet structure around $570 \mathrm{~s}$ in the 2006 WET run on GD 358 led us to suspect that oblique pulsation could be the cause. Fortunately, this hypothesis makes three directly testable predictions. We list these predictions along with our findings below:

I. The frequency of each $m$-component of a multiplet should be split into $2 \ell+1$ components which are exactly evenly spaced, with the spacing given by the surface rotation rate.

Finding: Assuming exactly equal spacing of two triplets (the first column in Table 1) we are able to produce an excellent pre-whitening of the data [see 4]. The derived 


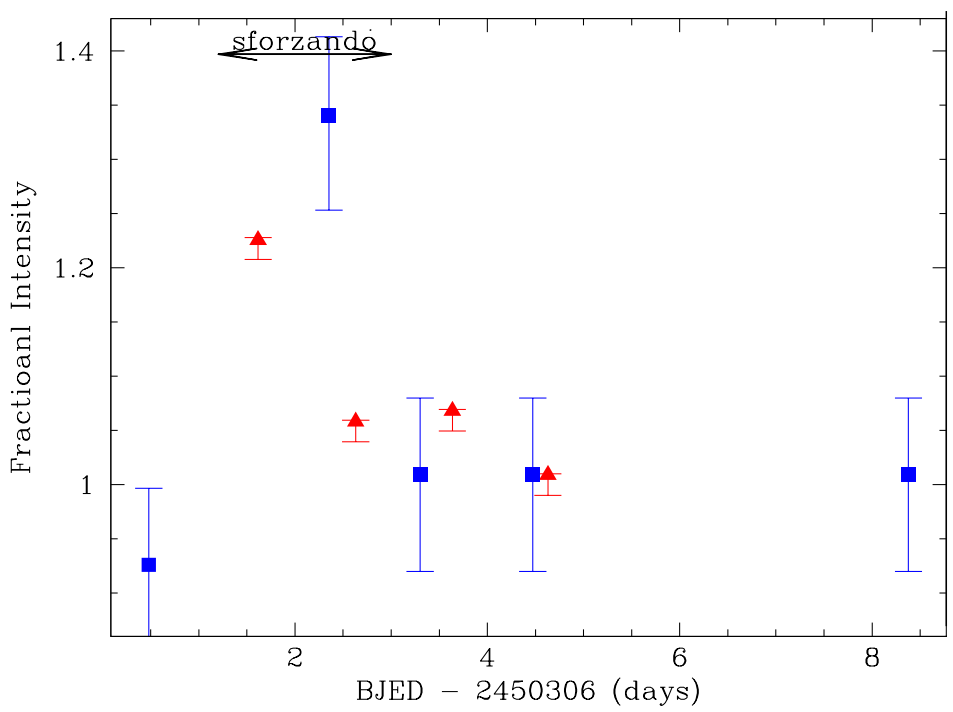

FIGURE 3. The relative intensity of GD 358 as measured relative to its levels after the sforzando episode. The triangles are data from McDonald Observatory and the squares are data from Mt. Suhora Observatory. Both data sets indicate a jump in intensity near BJED 2450311.

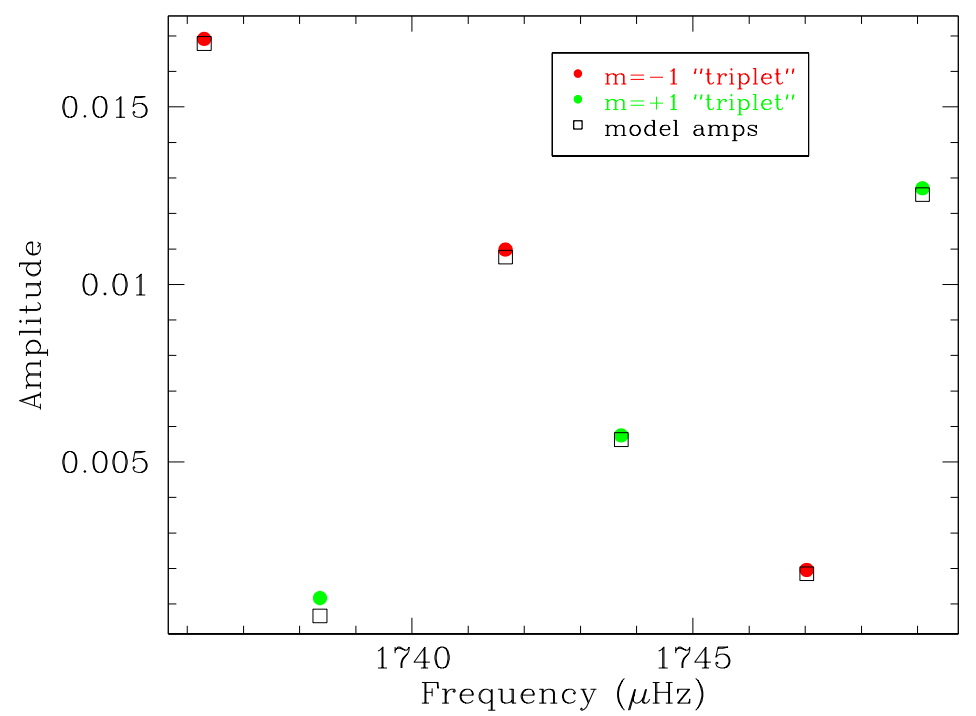

FIGURE 4. A fit of the calculated amplitudes (open squares) to the observed amplitudes (points) for the two triplet components given in Table 1 assuming the oblique pulsator model. The errors on the observed amplitudes are smaller than the size of the points. 
TABLE 1. Frequency solution for the oblique pulsation model:

$f_{\text {rot }}=5.362 \pm 0.003 \mu \mathrm{Hz}$

\begin{tabular}{lccc}
\hline Frequency $(\mu \mathbf{H z})$ & Amplitude (mma) & Phase (rad) & $\Delta \Phi / 2 \pi$ \\
\hline \multicolumn{4}{c}{ Triplet 1 } \\
\hline $1736.302 \pm 0.004$ & $16.77 \pm 0.13$ & $0.739 \pm 0.008$ \\
$1741.664 \pm 0.003$ & $10.81 \pm 0.13$ & $2.832 \pm 0.012$ & $0.510 \pm 0.013$ \\
$1747.027 \pm 0.004$ & $1.75 \pm 0.13$ & $1.724 \pm 0.075$ \\
\hline \multicolumn{5}{c}{ Triplet 2 } \\
\hline \multicolumn{5}{c}{$0.95 \pm 0.13$} & $1.268 \pm 0.138$ & \\
$1738.362 \pm 0.005$ & $5.56 \pm 0.13$ & $0.174 \pm 0.023$ & $0.517 \pm 0.023$ \\
$1743.725 \pm 0.003$ & $12.55 \pm 0.13$ & $2.117 \pm 0.011$ \\
$1749.087 \pm 0.005$ & & & \\
\hline
\end{tabular}

surface rotation rate is $f_{\text {rot }}=5.362 \pm 0.003 \mu \mathrm{Hz}$.

II. The phases of each of these split components must satisfy a given algebraic relation, namely $\Delta \Phi \equiv 2 \Phi_{0}-\left(\Phi_{+}+\Phi_{-}\right)$should be equal to either 0 (for an $m=0$ mode split by oblique pulsation) or $\pi$ (for $m= \pm 1$ modes split by oblique rotation), where $\Phi_{0}$ is the phase of the central component of each triplet and $\Phi_{ \pm}$refers to the phases of the high and low frequency components, respectively.

Finding: As shown in the last column of Table $1, \Delta \Phi=\pi$ to within the errors for both triplets, signaling that each triplet corresponds to an $|m|=1$ mode aligned with the pulsation axis, which, due to the mis-alignment of the pulsation and rotation axes is split into an equally spaced triplet.

III. The oblique pulsation model makes a prediction for the relative amplitudes within each of the triplets in Table 1. These expressions are given in, e.g., the appendix of Montgomery et al. [4].

Finding: The oblique pulsation model provides an excellent fit to the observed amplitudes within each triplet. Although the number of amplitudes (6) is only slightly larger than the number of free parameters (5), by randomly generating amplitudes for 500 pairs of triplets we found that only $2 \%$ of the time were better fits found than our current fit. We therefore conclude that our fits are significant at the $98 \%$ level. The derived parameters are: $\theta_{i}=46.3^{\circ}, \beta=31.8^{\circ}$, and $x_{1}=5.65$, where $\beta$ is the angle between the pulsation and rotation axes and $x_{1}$ is a measure of the strength of the magnetic to the rotational perturbations to the mode's frequency. We note that this value of $\theta_{i}$ is close to that obtained from our nonlinear light curve fits, $47.5 \pm 2.2^{\circ}$. This provides an important consistency check on both methods.

\section{CONCLUSIONS}

We have presented evidence that GD 358 is the first single white dwarf to show a shortlived, sudden temperature change and the first pulsating white dwarf to show clear signs of oblique pulsation. A critical component of these analyses was the nonlinear light curve fitting technique that we have developed. One of the long term goals of this fitting technique is to place stars in the $\tau_{0}-T_{\text {eff }}$ plane, as shown in Figure 5. 


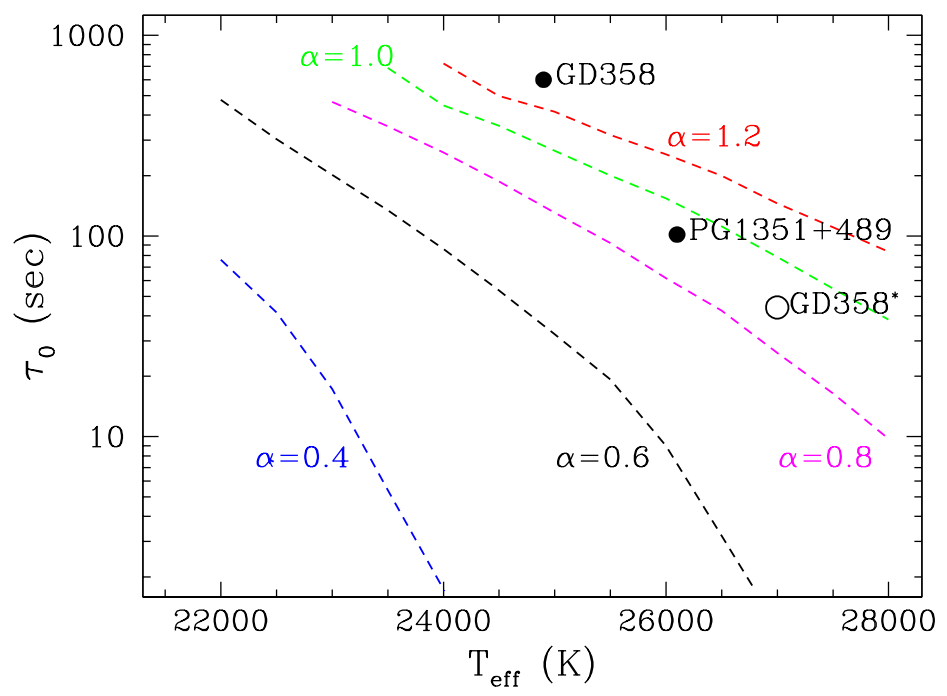

FIGURE 5. A comparison of the derived convective parameters $\tau_{0}$ with values expected from ML $2 / \alpha$ convection. The labeled points are individual objects and the dashed curves are the calculations. The label "GD 358" represents its state during the 2006 WET run while "GD 358*" denotes it during the sforzando.

\section{ACKNOWLEDGMENTS}

This work was supported by the National Science Foundation under grant AST-0909107, by the Norman Hackerman Advanced Research Program under grants 003658-02552007 and 003658-0252-2009, and by the Delaware Asteroseismic Research Center.

\section{REFERENCES}

1. D. E. Winget, E. L. Robinson, R. D. Nather, and G. Fontaine, ApJ 262, L11 (1982).

2. P. A. Bradley, and D. E. Winget, ApJ 430, 850 (1994).

3. D. E. Winget, R. E. Nather, J. C. Clemens, J. L. Provencal, S. J. Kleinman, P. A. Bradley, et al. ApJ 430, 839 (1994).

4. M. H. Montgomery, J. L. Provencal, A. Kanaan, A. S. Mukadam, S. E. Thompson, J. Dalessio, H. L. Shipman, D. E. Winget, S. O. Kepler, and D. Koester, ApJ 716, 84 (2010).

5. M. H. Montgomery, ApJ 633, 1142 (2005).

6. A. J. Brickhill, MNRAS 259, 519 (1992).

7. Y. Wu, MNRAS 323, 248 (2001).

8. M. H. Montgomery, in Astronomical Society of the Pacific Conference Series, edited by A. Napiwotzki, and M. R. Burleigh, 2007, vol. 372 of Astronomical Society of the Pacific Conference Series, p. 635 .

9. M. H. Montgomery, Communications in Asteroseismology 154, 38 (2008).

10. S. O. Kepler, R. E. Nather, D. E. Winget, A. Nitta, S. J. Kleinman, T. Metcalfe, et al., A\&A 401, 639 (2003).

11. J. L. Provencal, M. H. Montgomery, A. Kanaan, H. L. Shipman, D. Childers, A. Baran, ApJ 693, 564 (2009).

12. C. Weidner, and D. Koester, $A \& A$ 406, 657 (2003). 